\title{
Die integrationspolitischen Wirkungen der Römischen Verträge - Eine Bilanz nach 50 Jahren
}

\author{
Hanns Jürgen Küsters*
}

Für den europäischen Einigungsprozess bedeutete sie einen Quantensprung, die Unterzeichnung der Römischen Verträge am 25. März 1957. ${ }^{1}$ Mit Gründung der Europäischen Wirtschaftsgemeinschaft (EWG) und der Europäischen Atomgemeinschaft (EURATOM) signalisierten die sechs Montanunionstaaten ihre grundsätzliche politische Bereitschaft, den Weg der Integration auf supranationaler Ebene durch neue völkerrechtlich verbindliche Vereinbarungen fortzusetzen. Einen solchen Erfolg hatten die meisten westeuropäischen Politiker zweieinhalb Jahre zuvor kaum für möglich gehalten. Nach dem Debakel vom 30. August 1954, als der Vertrag über die Gründung der Europäischen Verteidigungsgemeinschaft und damit das Projekt einer Europäischen Politischen Gemeinschaft am Votum der französischen Nationalversammlung gescheitert war, standen die Sechs vor einem integrationspolitischen Scherbenhaufen. In einer besonderen historischen Konstellation kamen dann die Römischen Verträge zustande ${ }^{2}$ und bilden seitdem ein solides Fundament, auf dem die Europäische Union fest verankert steht. Um so berechtigter ist die Frage nach den langfristigen integrativen Wirkungen, die von den Verträgen auf die Gemeinschaftsentwicklung ausgingen. Woraus bestanden die Stützpfeiler, auf denen sich die europäische Einigung in den vergangenen fünf Jahrzehnten mühsam, aber dennoch kontinuierlich weiterentwickeln konnte?

\section{Integrationsmotive}

Gemeinsames außenpolitisches Hauptmotiv war die Westbindung der Bundesrepublik Deutschland. Nachdem das Problem ihrer sicherheitspolitischen Einbettung und kontrollierten Wiederbewaffnung durch Aufnahme in die Allianzen NATO und WEU gelöst war, galt es nun, die Westdeutschen auch wirtschaftlich noch stärker im Westen zu verankern und die zivile Kernenergienutzung voranzutreiben.

Weitere Haupttriebfeder waren ungelöste Außenwirtschaftsprobleme. In der ersten Dekade nach dem Zweiten Weltkrieg erfolgte der Handel unter den europäischen Staaten auf bilateraler Grundlage, indem entweder jede Seite sich bereit erklärte, zu einem festgelegten Wechselkurs die Währung des Partnerlandes bis zu einem vereinbarten Umfang gegen eigene Währung anzukaufen, oder Abkommen über die jeweils benötigten Warenlieferungen abgeschlossen wurden. Ohne die von der Regierung in Washington begonnene Marshallplanhilfe wäre die Wiederherstellung der europäischen Arbeitsteilung gewiss nicht gelungen. Im Kern ging es darum, den beteiligten europäischen Staaten Investitionsgüter zur Verfügung zu stellen, sie langfristig von Hilfslieferungen aus den Vereinigten Staaten von Amerika unabhängig zu machen und Westeuropa wieder auf eigene Füße zu bringen. Dazu mussten Handelshemmnisse abgebaut, freie Konvertibilität unter den europäischen Währungen hergestellt und

* Prof. Dr. Hanns Jürgen Küsters, apl. Professor für Wissenschaft von der Politik und Zeitgeschichte, Institut für Politische Wissenschaft und Soziologie, Universität Bonn.

1 Vertrag zur Gründung der Europäischen Wirtschaftsgemeinschaft, 25. März 1957, in: Bundesgesetzblatt (BGB1.) II 1957, S. 766-1013. Vertrag zur Gründung der Europäischen Atomgemeinschaft, 25. März 1957, ebenda, S. 1014-1223.

2 Franz Knipping: Rom, 25. März 1957. Die Einigung Europas, München 2004, S. 98-102. 
Zahlungsbilanzdefizite beseitigt werden. In der Organisation für Europäische Wirtschaftliche Zusammenarbeit (OEEC) kamen Liberalisierungsschritte aber nur mühsam zustande. Der innereuropäische Handel stagnierte, weil keine Möglichkeit bestand, die in europäischen Währungen erwirtschafteten Handelsbilanzüberschüsse in amerikanische Dollar zu konvertieren, was zu einem verstärkten zweiseitigen Tauschhandel führte. Durch Steigerung des innereuropäischen Handels sollte die Dollarlücke geschlossen werden. ${ }^{3}$ Die 1950 gegründete Europäische Zahlungsunion (EZU), ein Zusammenschluss mehrerer europäischer Länder zur Förderung des multilateralen Handels- und Zahlungsverkehrs, funktionierte durch einen Kreditmechanismus als multinationale Verrechnungsstelle, über die Überschüsse und Defizite saldiert und in Dollar ausgeglichen werden konnten. Erforderliches Kapital wurde aus dem Marshallplan zur Verfügung gestellt. Letztlich zielte die EZU auf die freie Konvertibilität aller beteiligten Währungen ab. Doch wurde der Außenhandel dadurch nur teilweise liberalisiert, zumal der EZU politische Sanktionsinstrumente fehlten. Nachdem im Dezember 1958 auf Initiative Großbritanniens 14 westeuropäische Staaten - darunter die sechs EWGStaaten - ihre Währungen für frei konvertierbar erklärten, wurde die EZU aufgelöst.

Für alle Mitgliedstaaten eröffneten sich mit den Römischen Verträgen neue Perspektiven. Innenpolitisch labil, war die vom chronischen Devisenmangel wegen kolonialer Verpflichtungen geplagte IV. Französische Republik gezwungen, protektionistisches Denken und das verkrustete System der ,Planification“ aufzugeben, um die eigene Wirtschaft zu modernisieren und internationale Wettbewerbsfähigkeit zu erlangen. Die junge Bundesrepublik Deutschland, gerade vom Besatzungsstatut befreit, war bei zehn Millionen zu integrierenden Vertriebenen und Flüchtlingen von Nahrungsmittel- und Rohstoffimporten abhängig. Kapital für den Wiederaufbau musste die deutsche Industrie durch Exportüberschüsse erwirtschaften. Das veranlasste die Bundesregierung, sich für die Handelsliberalisierung und freie Konvertibilität der Währungen einzusetzen. Italien, wo sich weite Regionen mehr auf dem Niveau eines fortgeschrittenen Entwicklungslandes befanden, als dass sie einem modernen, leistungsfähigen Industriestaat entsprachen, konnte von der neuen Wirtschaftsgemeinschaft nur profitieren. Ähnliche Interessen bewogen die kleineren überwiegend Agrargüter exportierenden Länder Belgien, Luxemburg und die Niederlande. Sie alle hatten im Zweiten Weltkrieg unter deutscher Besatzungsherrschaft gelitten und versprachen sich von der Bindung an die expandierende deutsche Wirtschaftskraft eigene Prosperitätsfortschritte.

Mit dem EWG-Vertrag vollzogen die Mitgliedstaaten einen Wechsel der Integrationsmethode. Sie sagten sich vom Konzept der additiven Teilintegration der Europäischen Kohleund Stahlgemeinschaft ${ }^{4}$ los und wandten nun den gesamtwirtschaftlichen Integrationsansatz an. Schon kurze Zeit nach dem Start der Montanunion 1952 waren unter den Experten erhebliche Zweifel laut geworden, ob der Sektorintegrationsansatz die geeignete Grundlage für die sukzessive Vergemeinschaftung weiterer Wirtschaftsbereiche wäre. Weder bestand Einigkeit darüber, welche Sektoren in welcher Intensität und mit welchen Kompetenzerweiterungen für die Gemeinschaftsinstitutionen hinzugefügt werden sollten - in Frage kamen die Bereiche Verkehr, Telekommunikation und Post, die ausnahmslos unter staatlicher Kontrolle standen beziehungsweise verstaatlicht waren -, noch herrschte Klarheit über die politischen Konsequenzen, wenn alle Verkehrsbereiche - Bahn, Straße, Luftverkehr - integriert würden. Die Verträge von Rom implizierten Grundsatzentscheidungen über die weiter zu

3 Helge Berger/Albrecht Ritschl: Die Rekonstruktion der Arbeitsteilung in Europa. Eine neue Sicht des Marshallplans in Deutschland 1947-1951, in: Vierteljahrshefte für Zeitgeschichte 3/1995, S. 473-519.

4 Dirk Spierenburg/Raymond Poidevin: Histoire de Haute Autorité de la Communauté Européenne du Charbon et de l'Acier, Brüssel 1993. 
verfolgende Integrationsmethode. Über den wirtschaftlichen Zusammenschluss sollte die politische Einigung vorangetrieben werden, in der Hoffnung, diese werde sich aufgrund wachsender Verflechtung irgendwann quasi von selbst ergeben.

Einen Gemeinsamen Markt mit dem Kern einer Zollunion zu errichten, der die Inkorporierung aller Wirtschaftsbereiche beabsichtigte, auf dem binnenmarktähnliche Verhältnisse herrschen sollten und supranational agierenden Institutionen dazu politische Kompetenzen verlieh, stellte ein Novum dar. Die Mitgliedstaaten sahen sich gezwungen, eine ökonomische Konsolidierung und Modernisierung ihrer Volkswirtschaften zu betreiben, die sie nationalstaatlich kaum mehr bewerkstelligen konnten. Das Konzept der Wirtschaftsintegration unter administrativer Eigenständigkeit der damals innovativen zivilen Atomenergienutzung behielt das Ziel einer Politischen Union im Auge.

Obwohl nach den Wirren des Zweiten Weltkriegs die Menschen in Frieden leben wollten, haben die Regierungen ihren Bevölkerungen die Integration letztlich verordnet. Weder die Montanunion noch die Römischen Verträge entsprangen einem durch Wahlen oder Referenden manifestierten Wunsch nach einem Zusammenschluss. Und erst recht nicht gingen die Gemeinschaftsgründungen auf Initiative föderalistischer Bewegungen zurück, wenngleich sie diese natürlich lebhaft begrüßten. Es handelte sich vielmehr um einen Prozess, der von wenigen europäisch gesinnten Politikern und Ministerialbeamten gesteuert wurde, die den Integrationsgedanken zu konkreten Ergebnissen führen wollten. Doch auch sie vermochten kaum einzuschätzen, wie viel integrationspolitische Substanz den Verträgen faktisch innewohnt. $\mathrm{Zu}$ weit lagen theoretische Anschauungen und praktische Erfahrungen auseinander.

\section{Herzstück europäischer Integration: Gemeinsamer Markt und Zollunion}

Zweifelsohne entwickelte sich die Idee des Gemeinsamen Marktes zur stärksten Triebund Anziehungskraft für den gesamten europäischen Integrationsprozess. Im Geltungsbereich des EWG-Vertrages Verhältnisse zu schaffen, wie sie auf den nationalen Märkten herrschen, war und ist das mit Abstand ehrgeizigste und zugleich wirkungsmächtigste Vorhaben zur Friedenssicherung, das im 20. Jahrhundert begonnen wurde.

Im Mittelpunkt standen zwei grundsätzliche Überlegungen: Die eine, mehr wirtschaftliche Absicht zielte auf den institutionell vereinbarten Abbau bestehender Beschränkungen im zwischenstaatlichen Wirtschaftsverkehr auf dem Territorium der Mitgliedstaaten einschließlich der Vereinbarung eines gemeinsamen Zolltarifs, einer gemeinsamen Handelspolitik gegenüber dritten Ländern und die Herstellung eines Systems, das vor Wettbewerbsverfälschungen schützt. Die andere, mehr politische Absicht sah den EWG-Vertrag als funktionalen Schritt, dem weitere konstitutive Akte folgen würden. In der Präambel bekundeten die Vertragsparteien ihre Bereitschaft, ,,die Grundlagen für einen immer engeren Zusammenschluss der europäischen Völker“" zu schaffen, ohne jedoch den angestrebten Endzustand näher zu beschreiben. Zugleich forderten sie „die anderen Völker Europas“ auf, „durch ihre Wirtschaftskräfte Frieden und Freiheit zu wahren“ und ,sich diesen Bestrebungen anzuschließen“. Damit war die für die Gemeinschaft geradezu schicksalhafte Symbiose geboren, die unverändert Gültigkeit besitzt: Vertiefung und Erweiterung als parallele Aufgaben einer fortschreitenden politischen Integration.

Konkrete Hinweise auf die Notwendigkeit eines Gemeinsamen Marktes tauchten erstmals in dem Vertragsentwurf über die Satzung der Europäischen Gemeinschaft von $1953^{5}$

5 Entwurf eines Vertrages über die Satzung der Europäischen Gemeinschaft, angenommen von der Ad-hoc-Versammlung, Straßburg, 10. März 1953, in: Europa, Dokumente zur Frage der Europäischen Einigung, herausgegeben im Auftrag des Auswärtigen Amtes, Zweiter Teilband, Bonn 1962, S. 947-976. 
auf. Artikel 82 Absatz 1 charakterisierte den Gemeinsamen Markt als „freien Umlauf der Güter und des Kapitals und der Freizügigkeit der Menschen“, der in Anwendung der Artikel 2 bis 4 des EGKS-Vertrages ,fortschreitend“ verwirklicht werden sollte. In zweierlei Hinsicht würde sich allerdings der künftige Gemeinsame Markt grundlegend von einer bloßen weitergehenden Verflechtung der beteiligten Volkswirtschaften unterscheiden: Einerseits sollten den freien Wettbewerb behindernde Zölle und sonstige diskriminierende Maßnahmen und Praktiken eliminiert werden; andererseits würde der Gemeinsame Markt nicht auf der Basis der Goldwährung aufgebaut werden wie vor den beiden Weltkriegen. Über erforderliche Schritte waren sich die Wirtschaftssachverständigen des Wissenschaftlichen Beirats beim Bundeswirtschaftsministerium weitgehend einig: sukzessive Beseitigung der Zölle im Inneren des Marktes, Harmonisierung der Außenzölle, volle Liberalisierung des Güter-, Kapital- und Dienstleistungsverkehrs, Freizügigkeit des Personenverkehrs, Verhinderung von Wettbewerbsverfälschungen und Verständigung über die Grundzüge des Wettbewerbs sowie freie Austauschbarkeit der Währungen und Stabilität der Austauschrelationen wurden als unabdingbare Voraussetzungen angesehen.

Daher beschreibt der EWG-Vertrag mit seinen 248 Artikeln zunächst die Grundregeln (Art. 1-8) und führt das Tableau künftiger Aufgaben sowie auszuführender Gemeinschaftstätigkeiten auf (Art. 2-3). Sie stellen nach wie vor das Herzstück des europäischen Integrationsprozesses dar. An deren oberster Stelle steht die Errichtung eines Gemeinsamen Marktes durch schrittweise Annäherung der Wirtschaftspolitik der Mitgliedstaaten. Vor allem ging und geht es darum, drei Ziele zu realisieren: die ,harmonische Entwicklung des Wirtschaftslebens" im Inneren der Gemeinschaft, eine permanente und ,,ausgewogene Wirtschaftsausweitung" sowie „größere Stabilität" und „beschleunigte Hebung der Lebenshaltung“ sicherzustellen. In Anbetracht der disparaten wirtschaftlichen Voraussetzungen so unterschiedlicher Staaten wie des rückständigen, allenfalls agrarwirtschaftlich leistungsfähigen Italiens, veralteter Industriestrukturen Frankreichs mit hohem Anteil in der Landwirtschaft Tätigen, der wachsenden deutschen Exportindustrie und den agrarexportabhängigen Beneluxstaaten bestand die eigentliche Herausforderung in der Herstellung wirtschaftlich stabiler Verhältnisse sowie in der Angleichung und stetigen Verbesserung des Lebensstandards unter den Gemeinschaftsmitgliedern. Dies waren Grundvoraussetzungen, die Integration politisch erfolgreich fortsetzen zu können. Mithin musste der Integrationsprozess so gesteuert werden, dass nach einer Übergangszeit einerseits Fortschritte in der Lebenshaltung spürbar waren und andererseits diese nicht zu Lasten oder auch zum Vorteil einzelner Mitgliedstaaten vollzogen wurden.

\section{Integrationspolitische Weichenstellung}

Die Entscheidung im EWG-Vertrag für das Zollunionskonzept und deren zügige Realisierung unwiderruflich mit einem Endzeitpunkt innerhalb von zwölf Jahren in drei Etappen zu je vier Jahren vertraglich zu fixieren (Art. 8), war zunächst die entscheidende Antriebskraft für alle weiteren Integrationsbemühungen und bedeutete gleichermaßen die Ablehnung des Konzepts einer Freihandelszone auf intergouvernementaler Ebene. Bekanntlich war die Entscheidung Zollunion oder Freihandelszone höchst umstritten. Die Zollunionsverfechter sahen darin gemäß GATT-Vertrag einen ersten Ansatz zu einem globalen Freihandelssystem. Dagegen fürchteten Verfechter der Freihandelszone, allen voran Bundeswirtschaftsminister Erhard, die Entwicklung eines Regionalismus mit stark protektionistischen Wirkungen, der Handelsdiskriminierungen Vorschub leiste. Angesichts der konzeptionellen Alternative, im Rahmen der OEEC unter Einschluss Großbritanniens eine rein industrielle 
Freihandelszone einzurichten, ${ }^{6}$ implizierte die Zollunionsgründung unter den Sechs eine qualitativ gravierende Richtungsentscheidung für den weiteren Einigungsprozess. Der Preis war die handelspolitische Zweiteilung Westeuropas in den 1960er Jahren.

Hätte sich die britische Regierung, die im Jahre 1956 wegen ihres Commonwealth-Präferenzsystems kein Interesse an Vereinbarungen über einen gleichen Außenzolltarif und einer gemeinsamen Agrarpolitik zeigte, zudem ihren Export beeinträchtigt und die Lebenshaltungskosten steigen sah, mit dem Freihandelskonzept durchgesetzt, wäre die EWG wohl kaum als einheitlicher Wirtschaftsraum so schnell zusammengewachsen. Denn der Zollabbau und strenge Wettbewerbsregeln waren auf Dauer durch das GATT und die OEEC nicht ausreichend zu gewährleisten. Dazu bedurfte es institutioneller Absicherungen, die im Rahmen einer Zollunion rigide umzusetzen waren. Schon allein die vertragliche Verpflichtung, spätestens bis zum Jahre 1970 Zölle und mengenmäßige Beschränkungen beim Import und Export von Gütern vollständig abzubauen, einen gemeinsamen Zolltarif und eine gemeinsame Handelspolitik gegenüber Drittländern einzuführen ${ }^{7}$ sowie Hindernisse für den freien Personen-, Dienstleistungs-, Güter- und Kapitalverkehr zwischen den Mitgliedstaaten zu beseitigen, löste nicht zu überschätzende integrative Effekte aus. Unter anderem deswegen, weil derlei Entscheidungen die im Rat agierenden Mitgliedstaaten, die Kommission und auch Regierungen derjenigen Staaten, die nicht EWG-Mitglied waren, unter Handlungszwang setzten. Dabei bewährte sich die semi-lineare Methode, der zufolge der Zollabbau bei bestimmten Waren von den Regierungen selbst gesteuert werden konnte, wobei sich allerdings an der Zeitabfolge der Herabsetzungen, wie sie schon im Spaak-Bericht ${ }^{8}$ vorgesehen waren, nichts änderte.

Gegen Ende der Brüsseler Vertragsverhandlungen im Februar 1957 knüpften die Sechs harte Bedingungen an das Angebot gegenüber Großbritannien, auf dem Wege der Freihandelszone eine möglichst enge Assoziierung mit den EWG-Ländern anzustreben. Die im EWG-Vertrag geregelten Fragen des Zollabbaus und der mengenmäßigen Beschränkungen, der gemeinsame Außentarif, die Einbeziehung der Agrarprodukte und das Schutzklauselsystem sollten unverändert erhalten bleiben. Damit würden die Handelsbeziehungen nicht auf eine klassische Freihandelszone beschränkt, sondern den Bedingungen des Gemeinsamen Marktes möglichst weit angenähert werden. Dissens war infolgedessen vorprogrammiert. De Gaulles Abbruch der Freihandelszonen-Verhandlungen im Herbst 1958 signalisierte die Bereitschaft der neuen V. Französischen Republik, den Verpflichtungen des EWG-Vertrages nachzukommen, und zwang die britische Regierung, eine Ersatzlösung in Form einer kleinen Freihandelszone durch Gründung der Europäischen Freihandelsassoziation (EFTA) in der Stockholmer Konvention vom 4. Januar 1960 zu präsentieren, die wenig später am 3. Mai 1960 in Kraft trat. Ihr gehörten mit Dänemark, Großbritannien, Norwegen, Österreich, Portugal, Schweden, Schweiz (damit indirekt Liechtenstein, aufgrund der Verbindung mit der Schweiz durch eine Zollunion) und ab Juni 1961 Finnland an, das durch ein Assoziationsabkommen in den territorialen Anwendungsbereich der EFTA einbezogen war.

Dem Ziel der EFTA, eine starke, selbständige, handelspolitische Verhandlungsposition gegenüber der EWG aufzubauen, waren nur kurzfristig Erfolge beschert. Zum 1. Juli 1960

6 OEEC, Council: Resolution Concerning the Study of the Relationship between the proposed European Customs Union and Member Countries not Taking Part therein, 21. Juli 1956, in: Negotiations for an European Free Trade Area, Cmnd. 641, Her Majesty's Stationery Office, London 1959, S. 7.

7 Wendy Asbeek Brusse: Tariffs, trade and European integration, 1947-1957, New York 1997.

8 Regierungsausschuss, eingesetzt von der Konferenz von Messina, Bericht der Delegationsleiter an die Außenminister, 21. April 1956, in: Jürgen Schwarz (Hrsg.): Der Aufbau Europas. Pläne und Dokumente 1945-1980, Bonn 1980, S. 277-334. 
kam die erste vertraglich vereinbarte Zollsenkung zustande, und die Binnenzölle wurden aufgrund eines beschleunigten Zeitplans bereits Ende 1966, drei Jahre früher als ursprünglich geplant, stufenweise abgeschafft. Doch die eigentliche Absicht, sich als alternatives Integrationsmodell mit eigener Handlungsfähigkeit und Anziehungskraft in Europa zu etablieren, scheiterte aus mehreren Gründen bereits in der ersten Hälfte der 1960er Jahre. Zum einen verzeichneten die sechs EWG-Mitgliedstaaten ein schnelleres Wirtschaftswachstum als die EFTA-Mitglieder, die sich fortan von bilateralen Verhandlungen mit der EWG größeren Nutzen versprachen. Zum anderen schwächte das Vereinigte Königreich - einst Vorreiter der EFTA - die Organisation, indem es aufgrund seiner handelspolitischen Isolierung von der kontinentalen Sechser-Gemeinschaft schon im August 1961 den offiziellen Beitrittsantrag zur EWG stellte. Dem schlossen sich Dänemark, Norwegen und das bis dahin nichtgebundene Irland an. Zudem beantragten die neutralen EFTA-Staaten Österreich, Schweden und Schweiz die Assoziierung mit der EWG, die bis 1962 alle Importquoten abgeschafft hatte. Dass bereits zum 1. Juli 1968, anderthalb Jahre vor dem vertraglich vereinbarten Zeitpunkt am 1. Januar 1970, die Binnenzölle wegfielen und die Zollunion vollendet war, auf Güter aus Drittländern nun EWG-weit einheitliche Zölle erhoben wurden und der grenzüberschreitende Freihandel möglich war, stärkte die Gemeinschaft der Sechs und schuf das Fundament für den weltweit größten Handelsraum. Mit dem Beitritt Finnlands, Österreichs und Schwedens zur Europäischen Union 1995 schrumpfte die EFTA auf die Mitgliedstaaten Island, Liechtenstein, Norwegen und die Schweiz zusammen. ${ }^{9}$ Gerade die beiden Letztgenannten sind trotz des explizit bekundeten Willens ihrer Regierungen, aufgrund mehrerer negativ verlaufender Referenden heute noch nicht Mitglieder der Europäischen Union.

\section{Vier Freiheiten und Gemeinschaftspolitiken}

Liberalisierungseffekte im Inneren der Gemeinschaft und eine Konsolidierung gemeinsamer Interessen gegenüber Nicht-EWG-Mitgliedern besaßen vor allem die Herstellung der , vier Freiheiten“. Neben der Freizügigkeit des Warenverkehrs (Art. 9-37) implizierte die Zollunion den freien Personen- und Dienstleistungsverkehr und die Aufhebung der Beschränkungen im Kapitalverkehr (Art. 48-84). Sich dazu vertraglich zu verpflichten, war die französische Regierung wegen ihres Mangels an Devisen nicht bereit gewesen. Immerhin kristallisierte sich im Vertrag eine Regelung heraus, die zunächst keine völlige Beseitigung aller Beschränkungen im Kapitalverkehr zwischen den Mitgliedstaaten und dritten Ländern vorschrieb. Daher blieb es zunächst nur bei dem allgemein formulierten Ziel einer schrittweisen Koordinierung der Devisenpolitik unter den Mitgliedstaaten (Art. 70).

Darüber hinaus erforderte der Aufbau des Gemeinsamen Marktes eine Reihe weiterer Maßnahmen, die zu einer künftigen gemeinsamen Politik der Gemeinschaft (Art. 85-130) führen sollten. Die Vertragsbestimmungen über die Verkehrspolitik (Art. 74-84), Wettbewerbsregeln (Art. 85-94) und die Handelspolitik (Art. 110-116) sahen ausdrücklich die Erarbeitung gemeinsamer Politiken vor, die zugleich eine Art Aktionsplan zur Vertiefung der Integration darstellten.

Gerade die Bundesregierung beabsichtigte, mit strikten Wettbewerbsregeln Interventionen der Staaten und Kartelle zu verhindern, womit sie hauptsächlich die Praxis der französischen Regierung im Auge hatte. So waren Vereinbarungen zwischen Unternehmen nicht generell untersagt. Der EWG-Vertrag erklärte diese aber insofern für nichtig, wenn sie geeignet waren, den Handel unter den Mitgliedstaaten zu beeinträchtigen oder sie eine Wettbe-

9 EFTA Sekretariat (Hrsg.): 40 Years of Free Trade. EFTA at a Glance, Genf 2001. 
werbsverfälschung bezweckten (Art. 85). Zudem wurde die Gründung von Monopolen nicht verboten, sondern lediglich die missbräuchliche Ausnutzung einer marktbeherrschenden Stellung (Art. 86). Im Prinzip waren sich die Sechs über das Verbot von Dumpingpraktiken einig (Art. 91), allerdings schieden sich die Geister an der Frage zulässiger Ausnahmefälle, unter welchen Voraussetzungen und Bedingungen staatliche Subventionen („Beihilfen“) erlaubt wären (Art. 92-93). Der Entschluss der Vertragsparteien, der Kommission weitreichende Vollmachten für die Beurteilung der Zulässigkeit der Beihilfen in jedem Einzelfall zuzusprechen, verlagerte das Problem auf kommunitäre Ebene (Art. 94), ohne es wirklich zu lösen und verursachte einen fortdauernden Streit über die Praktiken der Wettbewerbspolitik.

Die fortschreitende Entwicklung des Gemeinsamen Marktes bedingte eine weitergehende Koordinierung der Wirtschaftspolitik der beteiligten Staaten, doch war es nach dem gescheiterten Vertrag über die Gründung der Europäischen Verteidigungsgemeinschaft (EVG) illusorisch anzunehmen, es würde zu Vereinbarungen über solch weitreichende Eingriffe der Gemeinschaft in die Wirtschaftspolitik der Mitgliedstaaten kommen. Natürlich war allen Beteiligten schon vor Vertragsunterzeichnung ebenfalls bewusst, dass die Funktionsfähigkeit des Gemeinsamen Marktes auch ein Währungssystem bedingte, in dem sich nicht nur dem Preis- und Kostenniveau entsprechende Wechselkurse bildeten, sondern unter den beteiligten Mitgliedstaaten ein Zahlungsbilanzausgleich mit marktmäßigen Mitteln erfolgen würde und die Wechselkurse stabil blieben. Doch die französische Regierung wehrte sich, der Gemeinschaft Steuerungsinstrumente zur Verfügung zu stellen. Wiederum fürchteten die Deutschen, am Ende komme eine stark dirigistische Regelung heraus. Vertragliche Verpflichtungen zum Zahlungsbilanzausgleich empfanden die Mitgliedstaaten als Beeinträchtigung ihrer Hoheitsrechte. Maximum des Erreichbaren waren unverbindliche Verpflichtungen. Wirtschaftspolitik (Art. 103-116), insbesondere die Konjunkturpolitik (Art. 103), Maßnahmen zum Ausgleich der Zahlungsbilanzen (Art. 104-109) sowie Fragen der Währungspolitik und der Sozialpolitik (Art. 117-128) wurden zwar als Angelegenheiten ,gemeinsamen Interesses“ definiert. Doch erklärten sich die Vertragsparteien lediglich zur Koordinierung der Wirtschaftspolitik und engeren Zusammenarbeit mit den zuständigen Verwaltungsstellen sowie zur Behebung von Störungen im Gleichgewicht ihrer Zahlungsbilanzen bereit.

Angesichts nationaler Interessendivergenzen war absehbar, dass es eine Herkulesaufgabe werden würde, die Wirtschafts-, Währungs- und Sozialpolitik in gemeinsame Politiken überzuführen und die Integration in diesen Bereichen zu vertiefen. Hier spielten alle EWGStaaten auf Zeit und warteten auf einen späteren ,Spill-over'-Effekt. Bevor nicht die Zollunion aufgebaut war, schien ein derartiger Versuch wenig Erfolg versprechend. Erst auf der Haager Gipfelkonferenz erteilten die Staats- und Regierungschefs im Dezember 1969 den Auftrag, einen Plan für die Errichtung einer Währungsunion und einer einheitlichen Währung auszuarbeiten. Der Werner-Plan scheiterte Mitte der 1970er Jahre, weil sich die internationale Währungsordnung nach dem Zusammenbruch des Bretton-Wood-Systems im Umbruch befand und ein Streit zwischen Ökonomisten und Monetaristen über die Frage entbrannte, ob zuerst die Wirtschaftspolitik oder zunächst die Währungen zu koordinieren seien. Dem 1979 eingerichteten Europäischen Währungssystem folgte dann auf der Grundlage des Delors-Plans am 1. Juli 1990 der Beginn der ersten Stufe zur Europäischen Wirtschafts- und Währungsunion.

Wie wenig ökonomische Auswirkungen der Integration tatsächlich vorherzusehen waren, zeigt die deutsch-französische Kontroverse über die Harmonisierung der Soziallasten. Sie hätte im Oktober 1956 die Brüsseler Vertragsverhandlungen fast zum Scheitern gebracht. Wollte Frankreich einerseits den beteiligten Regierungen allgemein weitgehende Hand- 
lungsfreiheit bei ihrer Wirtschaftspolitik belassen, forderte es andererseits die Arbeitsbedingungen - Löhne, Arbeitszeit, Urlaubsvorschriften - anzugleichen und einen Anpassungsund Investitionsfonds einzurichten. Das hatte die Bundesregierung abgelehnt, weil sie darin einen Eingriff in den Wettbewerb sah. Beim Aufbau der Zollunion spielte die angeblich so existenzielle Frage jedoch keine zentrale Rolle mehr.

Insgesamt zeigte sich: Je sensitiver der jeweilige Politikbereich war, desto schwieriger wurde die vertragliche Kodifizierung von gemeinsamen Politiken, desto größer blieb der integrative Handlungsbedarf. In den Politikbereichen, in denen der EWG-Vertrag nur wenig konkrete Maßnahmen beinhaltete, war das zu erwartende Konfliktpotenzial bei der Vereinbarung gemeinsamer Politiken umso größer und umgekehrt die Substanz für den weiteren Integrationsprozess umso geringer.

\section{Wunder Punkt: Landwirtschaftspolitik}

Von strikten Wettbewerbsbestimmungen des Gemeinsamen Marktes ausgenommen wurde die Landwirtschaft und der Handel mit landwirtschaftlichen Erzeugnissen (Art. 3847), für den der EWG-Vertrag ebenfalls die Gestaltung einer gemeinsamen Agrarpolitik verfügte (Art. 39). Angesichts der über viele Jahrzehnte von allen Mitgliedsländern praktizierten staatlichen Interventionspolitik beim Agrarhandel überraschte es nicht, dass die sechs Regierungen nur unter bestimmten Garantien einer Übertragung von Hoheitsrechten auf die Gemeinschaft zustimmten. Sicherung des Einkommens der in der Landwirtschaft Erwerbstätigen, die ein erhebliches Wählerpotenzial ausmachten, die verbreitete Denkweise, vornehmste Aufgabe des Staates sei es, die Nahrungsmittelversorgung der Bürger und die Unabhängigkeit von Nahrungsmittelimporten zu gewährleisten sowie der damalige Nachfrageüberhang gegenüber dem Angebot an Agrarprodukten spielten eine erhebliche Rolle.

Vertragsregelungen über die Landwirtschaft fielen deshalb so kompliziert aus, weil sie verschiedensten Interessen gerecht werden mussten. Die französische Regierung hatte insistiert, die gemeinsame Landwirtschaftspolitik darauf auszurichten, Agrarerzeugnisse zu den optimalen Bedingungen hinsichtlich des Ertrages und des Preises zu produzieren und den landwirtschaftlich Tätigen durch Steigerung des Pro-Kopf-Einkommens einen ausreichenden Lebensstandard zu sichern. Außerdem sollte beim Warenverkehr innerhalb der Gemeinschaft ein Mindestpreissystem zugelassen werden, das die freie Einfuhr von Agrarprodukten gestattete, soweit die Preise auf dem Binnenmarkt nicht unter ein bestimmtes Niveau fallen würden. Ferner wollte Frankreich langfristige vertragliche Verpflichtungen unter den Mitgliedstaaten für den Handel mit Agrarerzeugnissen aus den Überseegebieten vereinbaren, im Warenverkehr mit Drittländern ein Präferenzsystem einrichten, das den Absatz von Überschüssen und den Ausgleich von Defiziten der Gemeinschaft sicherstellte und schließlich ein Schutzsystem schaffen, das die Erhebung von Ausgleichzöllen zuließ, wobei auf einen Garantie- und Ausrichtungsfonds zurückgegriffen werden sollte. Während in der Bundesregierung das Bundeswirtschaftsministerium zumindest teilweise einen stärkeren internationalen Konkurrenzdruck auf die deutsche Landwirtschaft befürwortete, wehrte sich das Landwirtschaftsministerium mit Unterstützung des Deutschen Bauernverbandes gegen jede Preisgabe des Marktschutzes, weil beide die Existenz der deutschen Landwirte gefährdet sahen. Auch die deutsche Delegation trat dann für die Aufrechterhaltung stärkerer protektionistischer Regelungen ein.

Die eigentliche Schwierigkeit lag darin, die Öffnung der nationalen Agrarmärkte im Inneren der Gemeinschaft herzustellen und gleichzeitig einen gewissen nationalen Schutz der Landwirtschaften gegenüber anderen Mitgliedsländern wie gegenüber Drittstaaten so in Ein- 
klang zu bringen, dass die Dynamik des Einigungsprozesses in Zukunft nicht gehemmt würde. Im Wesentlichen ging es darum, vier Probleme zu lösen:

Die Aufgabe der einzelstaatlichen Marktordnungen zugunsten einer gemeinsamen Agrarpolitik (Art. 43): Die meisten Delegationen waren bereit, eine allmähliche Kompetenzübertragung von der nationalen auf die europäische Ebene am Ende der Übergangszeit zu akzeptieren, vorher allenfalls in Ausnahmefällen und dann zeitlich begrenzt.

Die Mindestpreise (Art. 44): Da kein Verhandlungspartner die Landwirtschaft direkt dem internationalen Wettbewerb aussetzen wollte, einigte man sich auf einen fortschreitenden Abbau der Zölle und mengenmäßigen Beschränkungen, der unter bestimmten Voraussetzungen die Festsetzung von Mindestpreisen erforderlich machte.

Langfristige multilaterale Abkommen (Art. 45): Alle Mitgliedstaaten waren daran interessiert, die Entwicklung des europäischen Agrarhandels durch den Abschluss multilateraler Verträge langfristig zu stabilisieren, bis die einzelstaatlichen Marktordnungen durch eine europäische Ordnung ersetzt würden.

Die Ausfuhrbeihilfen (Art. 45): Während der Übergangszeit und auch in der Endphase sollte der Agrarexport in Drittländer subventioniert werden, wenn die Rohstoffpreise höher lägen als die Weltmarktpreise. Für aus Drittländern importierte Rohstoffe sollten den Exporteuren der verarbeiteten Produkte Zölle, Umlagen und Einfuhrkosten erstattet werden. Die finanziellen Lasten wären nach Ablauf der Übergangszeit von der Gemeinschaft zu tragen.

Im Interesse aller legte der EWG-Vertrag das Instrumentarium fest und nannte die Ziele der gemeinsamen Agrarpolitik (Art. 38) - Steigerung der Produktivität, Gewährleistung einer angemessenen Lebenshaltung der landwirtschaftlich tätigen Bevölkerung, Stabilisierung der Märkte, Sicherstellung der Versorgung, Belieferung der Verbraucher zu angemessenen Preisen -, ließ aber die Inhalte zur Erreichung der Ziele offen.

Auf Vorschlag der EWG-Kommission trat ab 1962 die gemeinsame Agrarpolitik sukzessive in Kraft. ${ }^{10}$ Damit verordnete sie einen freien Markt der Agrarerzeugnisse innerhalb der EWG, erkannte die bäuerlichen Strukturen und natürlichen regionalen Unterschiede der Landwirtschaft in den Mitgliedstaaten an und strebte eine allmähliche Anpassungen der Verhältnisse an. Durch die Einführung von Präferenzregelungen zugunsten von EWG-intern erzeugten Produkten bei der Vermarktung und einer Preisvorteilsgarantie gegenüber importierten Agrarprodukten aus Drittländern betrieb die Kommission gemeinsam mit den Mitgliedsregierungen eine protektionistische Politik mit hohem Finanzaufwand, die den europäischen Landwirten genügend Einkommen garantierte, Konkurrenz von den Produkten aus Drittländern vermied und den Interessengruppen der Mitgliedstaaten entgegenkam.

\section{Von der Hilfe für koloniale Überseegebiete zur Entwicklungshilfepolitik}

Anders verhielt es sich mit der von der französischen Regierung in den Vertragsverhandlungen erhobenen Forderung nach Einbeziehung ihrer Überseegebiete in den Gemeinsamen Markt (Art. 131-136). Das Mutterland wollte damit koloniale Finanzlasten auf die europäischen Nachbarländer abwälzen. Die Zusage Adenauers, die er Ministerpräsident Mollet im Februar 1957 gab, einen deutschen Beitrag in gleicher Höhe wie Frankreich zu dem Entwicklungsfonds für die überseeischen Länder und Hoheitsgebiete jeweils mit insgesamt 200 Millionen EZU-Verrechnungseinheiten in fünf Jahren zu leisten (Durchführungsabkommen Anlage A), eröffnete der deutschen Wirtschaft Zugang zu Überseemärkten, vorwiegend in Afrika. Schließlich begründete diese Vereinbarung das entwicklungspolitische Engagement der

10 Hans von der Groeben: Aufbaujahre der Europäischen Gemeinschaft, Baden-Baden 1982, S. 146-156. 
EWG. Daraus resultierte das 1963 unterzeichnete Assoziierungsabkommen der EWG mit 17 afrikanischen Staaten und Madagaskar in Jaunde (Kamerun), dem 1969 das zweite JaundeAbkommen folgte. Beide waren Vorläufer der späteren Abkommen Lomé I-IV und des im Juni 2000 in Cotonou (Benin) unterzeichneten Abkommens zwischen der Europäischen Union und den Staaten Afrikas, der Karibik und des Pazifiks (den AKP-Staaten). Die integrationspolitische Bedeutung lag zum einen darin, dass erstmals die Sechs Finanzleistungen gegenüber den Kolonien der Mitgliedstaaten aufnahmen und somit Entwicklungshilfe zu einem Gemeinschaftsanliegen machten. Zum anderen tat die EWG damit im begrenzten Rahmen erste Schritte in Richtung einer gemeinsamen Außenpolitik gegenüber Staaten der Dritten Welt, aus der sich die heute weltweit größte koordinierte Entwicklungshilfe herauskristallisierte.

Dem Zugeständnis der Deutschen, die neben Frankreich größte Nettozahler waren, ohne selbst davon zu profitieren, stand die Zusage der übrigen Verhandlungsteilnehmer gegenüber, die Protokollerklärung hinzunehmen, dass mit der Schaffung des Gemeinsamen Marktes durch Deutschland keine Zollgrenze gezogen wurde und der innerdeutsche Handel künftig nicht als Außenhandel der EWG zu betrachten sei. Ein Grundsatzdokument, das für die nächsten 33 Jahre das Verhältnis der DDR zur Gemeinschaft bestimmte und sich im Zuge der Verhandlungen über die Wiederherstellung der deutschen Einheit 1990 als wichtiger Rahmen für die Eingliederung der DDR in die Europäischen Gemeinschaften erwies.

\section{Institutionelles System und Demokratiedefizite}

Der EWG-Vertrag etablierte ein sehr differenziertes Entscheidungssystem unter den Gemeinschaftsorganen (Art. 137-139), das gerade wegen seiner hohen Komplexität und sachlichen Differenzierung auch hinsichtlich der finanziellen Maßnahmen (Art. 193-209) für eine Ausgewogenheit bei der Durchsetzung von Gemeinschaftszielen und einzelnen Regierungsinteressen gesorgt hat. Das duale System aus dem Zusammenwirken von Kommission und Rat in einem Netzwerk komplizierter Entscheidungs- und Abstimmungsprozesse wurde einerseits dem Interesse der Regierungen gerecht, letzte Entscheidungs- und Gesetzgebungskompetenzen zu behalten, und andererseits entsprach es der Notwendigkeit, die Anwendung der Vertragsbestimmungen durch ein unabhängiges Organ auf kommunitärer Ebene sicherzustellen, welches das Gemeinschaftsinteresse repräsentiert. Der EWG-Vertrag beinhaltet daher intergouvernementale und supranationale Vorschriften, die ohne den Willen aller Beteiligten keine substanzielle Fortentwicklung der Integration erlauben. Die Stimmengewichtung bei Entscheidungen im Rat ermöglicht überdies eine austarierte nationale Interessenvertretung. Zugleich lassen die Vertragsbestimmungen den Mitgliedstaaten freie Hand, dynamisch die Integration in Richtung eines Bundesstaates oder in eine andere Form von Staatenverbindungen weiterzuentwickeln. Diese Offenheit impliziert Stärke und Schwäche gleichermaßen: Schwäche, weil das anzustrebende politische Ziel der Integration unbestimmt blieb; Stärke, weil alle institutionellen Instrumente vorhanden waren, erforderliche integrationspolitische Entscheidungen zu treffen.

Von Beginn an enthielten die Römischen Verträge jedoch ein Demokratiedefizit. Alle Beteiligten hatten zunächst absichtlich auf eine direkte demokratische Legitimation der Versammlung durch die europäischen Wähler verzichtet. Zum einen befürchteten sie Widerstände der nationalen Parlamente - nicht nur aus der französischen Nationalversammlung -, wenn durch Kompetenzerweiterung der Versammlung die Macht der Parlamente beschnitten und möglicherweise die Verträge von ihnen nicht ratifiziert und dem europäischen Integrationsprozess das endgültige Aus bescheren würden. Zum anderen gingen sie davon aus, dass aufgrund der sich allmählich vertiefenden Integration Kompetenzübertragungen in absehbarer Zeit von selbst thematisiert würden. Überschätzt wurde damals gewiss die Mög- 
lichkeit der nationalen Legislativen, maßgebliche parlamentarische Kontrolle über die Gemeinschaftspolitiken ausüben zu können. So besaß die Versammlung von allen Organen die mit Abstand schlechtesten Voraussetzungen, ihre Kompetenzen im Zuge des fortschreitenden Zusammenschlusses auszuweiten. Daran änderte auch die Umbenennung in Europäisches Parlament 1964 nichts. Die Integration war eben zuerst als ein gouvernementaler Prozess angelegt. Allein der Europäische Gerichtshof wahrte seine Position als Hüter der Verträge aller drei Gemeinschaften und wurde sich zunehmend seiner Bedeutung bewusst.

Der am 1. Juli 1967 wirksam gewordene Fusionsvertrag ${ }^{11}$ verschmolz die Exekutivorgane der EGKS, EWG und EURATOM und ersetzte damit die drei (Minister-)Räte sowie die beiden Kommissionen (EWG, EURATOM) und die Hohe Behörde (EGKS) durch je ein Organ, deren Tätigkeit sich aber nach den Bestimmungen der jeweiligen Gemeinschaft richtet. Durch die Fusion der Gemeinschaftsorgane wurden die Demokratiedefizite keineswegs verbessert. Grund für die Zusammenlegungen war neben der Straffung der Entscheidungsprozesse vor allem die Einführung eines gemeinschaftlichen Verwaltungshaushalts. Denn die EWG würde die Hauptlast des Integrationsprozesses tragen, und getrennte Entscheidungsgremien der Gemeinschaften erschwerten nur künftige Erweiterungsverhandlungen.

\section{EURATOM-Vertrag: ,Atome für den Frieden“}

Neben dem EWG-Vertrag stellt der EURATOM-Vertrag das zweite Standbein der Römischen Verträge dar, dessen integrationspolitische Bedeutung wegen des sektoralen Zusammenschlusses heute zumeist gering veranschlagt wird. Sah die Bundesregierung im EWGVertrag das eigentliche Kernelement der europäischen Integration, so erblickte die französische Regierung im EURATOM-Vertrag den zentralen Pfeiler. Neben der EWG eine Vertragsgemeinschaft für Kernenergie zu bilden, erschien aus mehreren Überlegungen sinnvoll. Einerseits galt es, dem Mangel an ,herkömmlichen ' Energieträgern abzuhelfen, andererseits galt Kernenergie in den 1950er Jahren als Energieträger der Zukunft, weil er Unabhängigkeit in der Energieversorgung verhieß und deren Investitionskosten die Finanzierungsmöglichkeiten der einzelnen Staaten überschritten hätte. Wegen ziviler und militärischer Verwendungsmöglichkeiten waren zudem besondere politische Rahmenbedingungen erforderlich, die eine möglichst lückenlose Überwachung der Nutzung erlaubten. An beiden hatte die Regierung in Paris wegen ihrer Ambitionen im Hinblick auf den Bau einer eigenen Atombombe und der gemeinschaftlichen Kontrolle der zivilen Kernenergienutzung gegenüber den Deutschen größeres Interesse als an dem Gemeinsamen Markt.

Das Projekt eines EURATOM-Vertrages kam also in erster Linie aufgrund des Interesses der französischen Regierung zustande, Zugang zu spaltbarem Material für die Nutzung der Kernenergie zu erlangen. Erste Vertragsentwürfe enthielten diskriminierende Regelungen für die Bundesrepublik Deutschland. Geplant war, dass EURATOM ein Einkaufs- und Verkaufsmonopol für spaltbares Material erhalten sollte, mit dem Hintergedanken, wirksame Kontrollen durchführen zu können. Die deutsche Atomindustrie wäre damit vollends von ausländischer Versorgung abhängig gewesen. Stattdessen hatte die Bundesregierung für den Kauf und Verkauf auch durch andere Stellen plädiert, weil es letztlich nur um die Verwendungskontrolle ginge. ${ }^{12}$ Besonders Bundesatomminister Strauß forderte die ausreichende und Deutschland nicht diskriminierende Versorgung und Verteilung sowie die Kontrolle aller Beteiligten über die Verwendung von Kernbrennstoffen. Ohnedies hatte die junge expandierende deutsche Atomindustrie keine Entwicklungschancen. Diese Forderung setzte die Bundesregierung durch.

11 Amtsblatt der Europäischen Gemeinschaften 152 vom 13. Juli 1967.

12 Peter Weilemann: Die Anfänge der Europäischen Atomgemeinschaft, Baden-Baden 1983, S. 76-101. 
Im Wesentlichen erlegte der EURATOM-Vertrag mit seinen 225 Artikeln der Atomgemeinschaft sechs Aufgaben auf: (1) Schaffung eines Gemeinsamen Marktes für kerntechnische Stoffe (spaltbare Stoffe, Ausgangsstoffe und Erze, aus denen die Ausgangsstoffe gewonnen werden) mit gemeinschaftlichem Eigentumsrecht, (2) Zusammenführung dazu bestimmter Rechtspersonen, nämlich Mitgliedstaaten, natürliche Personen sowie öffentliche oder private Unternehmen und Einrichtungen, (3) einen Beitrag zur Bildung und Entwicklung von Kernindustrien zu leisten, (4) Teilhabe aller Mitgliedstaaten am zivilen Nutzen aus der Entwicklung der Kernenergie, Versorgungssicherheit und technische Sicherheit zu gewährleisten, (5) zu verhindern, dass von Kernmaterial, dessen Nutzung zivilen Zwecken dienen soll, für militärische Zwecke abgezweigt wird, sowie (6) Sorge für die Erarbeitung und Anwendung einheitlicher Sicherheitsnormen beim Gesundheitsschutz der Bevölkerung und der Arbeitskräfte zu tragen. Außerdem stellt der Vertrag Fördermittel für Forschungs- und Investitionsvorhaben bereit und schafft eine Versorgungsagentur und eigene Entscheidungsorgane.

Durch den EURATOM-Vertrag wurde ein strenges Kontrollsystem eingeführt, dass der Gemeinschaft die ausschließliche Kompetenz übertrug, um der Nutzung von Kernmaterial für nicht zivile Zwecke durch die Mitgliedstaaten vorzubeugen. Gemeinschaftspolitiken in den Bereichen Umweltschutz und Forschung haben normgebend auf den europäischen Kernenergiesektor gewirkt. Das ändert jedoch nichts an der Tatsache, dass die Mitgliedstaaten bei der gemeinsamen Förderung der Kernenergie in der Folgezeit und heute noch unterschiedliche Strategien zur Gewährleistung ihrer Versorgungssicherheit anwenden. Nach den Unfällen von Three Mile Island und der Katastrophe von Tschernobyl entschlossen sich Italien und Belgien 1987, wegen der Risiken und möglicher Folgen eines atomaren Unfalls auf die Nutzung von Kernkraftwerken zur Energiegewinnung zu verzichten. Die in den 1990er Jahren beigetretenen Länder Österreich und Schweden hatten diesen Schritt schon 1978 beziehungsweise 1980 vollzogen. Während Dänemark, Griechenland und Irland den Bau neuer Kernkraftwerke stoppten, die Niederlande und Spanien per Gesetze den Bau untersagten und Deutschland im Jahr 2002 mit dem neuen Atomgesetz die Ausstiegsvereinbarung von 2000 rechtverbindlich umsetzte, hält Frankreich an der Kernkraftnutzung fest.

Die integrative Wirkung des EURATOM-Vertrages war gering, weil bisher eine Fusion von EWG und EURATOM unterblieb, obwohl sie gemeinsame Organe besitzen. Vielmehr haben beide ihre Rechtspersönlichkeit behalten. Zudem übertrug der EURATOM-Vertrag der Gemeinschaft kaum ausschließliche Zuständigkeit. So war es jedem Mitgliedstaat erlaubt, geeignete Rechts- und Verwaltungsvorschriften zu erlassen. Dabei blieb die Arbeit der EURATOM-Kommission und nationales Regierungshandeln im EURATOM-Rat weitgehend parlamentarischer Kontrolle entzogen. Auch gegenwärtig verfügt das Europäische Parlament weder beim Abschluss internationaler Atomverträge noch bei der Kreditvergabe im EURATOM-Rahmen über Mitbestimmungsrechte.

\section{Fazit}

Der EWG-Vertrag mit den Kernelementen Gemeinsamer Markt und Zollunion etablierte das Fundament der europäischen Integration und ist nach wie vor die tragende supranationale Säule der Europäischen Union. Die zügige Vollendung der Zollunion 1968 ermöglichte Ende 1969 weitere politische Entscheidungen sowohl zur ersten Erweiterung der Europäischen Gemeinschaft durch Aufnahme Großbritanniens, Dänemarks, Irlands und ursprünglich Norwegens als auch zur Vertiefung der Einigung durch Schaffung der Europäischen Politischen Zusammenarbeit und ersten konkreten Plänen zu einer Wirtschafts- und 
Währungsunion. Den Gemeinsamen Markt zu vollenden, bis wirklich binnenmarktähnliche Verhältnisse in allen Mitgliedstaaten herrschen, ist angesichts der ständig wachsenden Zahl der Gemeinschaftsmitglieder zu einer permanenten Herausforderung geworden.

Angesichts der Ausgangslage Mitte der 1950er Jahre war die sukzessive Entwicklung von Gemeinschaftspolitiken der richtige Weg. Dass die Mitgliedstaaten anfangs dem Landwirtschaftssektor nicht sofort harte Wettbewerbsbedingungen auferlegten, stellte a priori kein Integrationsmanko dar. Vielmehr war es dem politischen Kalkül der Regierungen der Mitgliedstaaten anzulasten, die stets auch nationalen Interessen Rechnung trugen und sich in den 1960er Jahren nicht auf einen harten Wettbewerbskurs in der Agrarpolitik verständigen konnten. Dies traf auch für das Problem der Mehrheitsentscheidungen im Rat zu, die der EWG-Vertrag beim Eintritt in die dritte Aufbauphase der Zollunion bestimmte, wogegen Frankreich sich wandte und 1966 den Luxemburger Kompromiss erzwang.

Trotz der ungewissen politischen Finalität der Einigung, die weder der EWG- noch der EURATOM-Vertrag zu klären vermochte, hat in den letzten 50 Jahren die strikte, institutionell verankerte Handelsliberalisierung als Frieden stiftender Ansatz zu den erwarteten politischen Stabilisierungseffekten in Europa geführt, wenn auch nicht zu einem europäischen Bundesstaat, so doch zu der Europäischen Union mit dem weltweit größten ökonomischen Potenzial und beharrlicher Anziehungskraft.

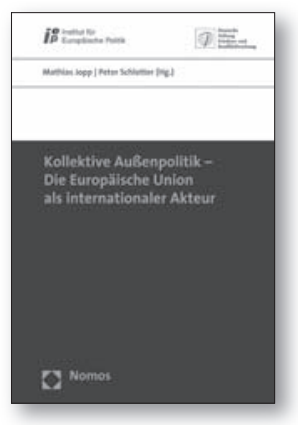

\section{Kollektive Außenpolitik - Die Europäische Union als internationaler Akteur}

Herausgegeben von Mathias Jopp und Peter Schlotter

2007, ca. 396 S., brosch., ca. 59,- $€$, ISBN 978-3-8329-2910-7

(Europäische Schriften, Bd. 86)

Erscheint August 2007

Bitte bestellen Sie bei Ihrer Buchhandlung oder bei Nomos | Telefon 07221/2104-37 | Fax -43 www.nomos.de | sabine.horn@nomos.de

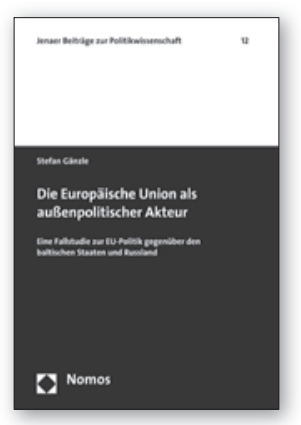

\section{Die Europäische Union als außenpolitischer Akteur}

Eine Fallstudie der EU-Politik gegenüber den baltischen Staaten und Russland

Von Stefan Gänzle

2007, 320 S., brosch., 54,- $€$, ISBN 978-3-8329-2285-6

(Jenaer Beiträge zur Politikwissenschaft, $B d .12)$

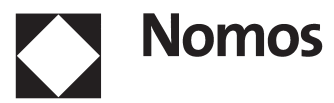

